\title{
Spuren des Denkens. Die Stellung einer Theologie der Septuaginta innerhalb der wissenschaftlichen Theologie
}

\begin{tabular}{|c|c|}
\hline \multicolumn{2}{|c|}{$\begin{array}{l}\text { Author: } \\
\text { Szabolcs-Ferencz Kató } 1,2\end{array}$} \\
\hline \multicolumn{2}{|c|}{$\begin{array}{l}\text { Affiliations: } \\
\text { }{ }^{1} \text { Department of Old } \\
\text { Testament and Hebrew } \\
\text { Scriptures, Faculty } \\
\text { of Theology and Religion, } \\
\text { University of Pretoria, } \\
\text { Pretoria, South Africa }\end{array}$} \\
\hline \multicolumn{2}{|c|}{$\begin{array}{l}\text { 2Department of Old Testament, } \\
\text { Protestant Theological } \\
\text { Institute of Cluj-Napoca, Cluj } \\
\text { Napoca, Romania }\end{array}$} \\
\hline \multicolumn{2}{|c|}{$\begin{array}{l}\text { Research Project Registration: } \\
\text { Project Leader: A. Groenewald } \\
\text { Project Number: } 02428024\end{array}$} \\
\hline \multicolumn{2}{|c|}{$\begin{array}{l}\text { Description: } \\
\text { This research is part of the } \\
\text { project, 'Prophetic Studies', } \\
\text { directed by Prof. Dr Alphonso } \\
\text { Groenewald, Department of } \\
\text { Old Testament Studies, } \\
\text { Faculty of Theology, } \\
\text { University of Pretoria. }\end{array}$} \\
\hline \multicolumn{2}{|c|}{$\begin{array}{l}\text { Corresponding author: } \\
\text { Szabolcs-Ferencz Kató, } \\
\text { katoszabolcs@yahoo.com }\end{array}$} \\
\hline $\begin{array}{l}\text { Dates: } \\
\text { Received: } 30 \text { I } \\
\text { Accepted: } 17 \\
\text { Published: } 14\end{array}$ & $\begin{array}{l}\text { May } 2020 \\
\text { Aug. } 2020 \\
\text { Oct. } 2020\end{array}$ \\
\hline \multicolumn{2}{|c|}{$\begin{array}{l}\text { How to cite this article: } \\
\text { Kató, S.-F., 2020, 'Spuren des } \\
\text { Denkens. Die Stellung einer } \\
\text { Theologie der Septuaginta } \\
\text { innerhalb der wissenschaftlichen } \\
\text { Theologie', HTS Teologiese } \\
\text { Studies/Theological Studies } \\
\text { 76(4), a6173. https://doi. } \\
\text { org/10.4102/hts.v76i4.6173 }\end{array}$} \\
\hline $\begin{array}{l}\text { Copyright: } \\
\text { @ 2020. The A } \\
\text { Licensee: AOS } \\
\text { licensed unde } \\
\text { Commons Att! }\end{array}$ & $\begin{array}{l}\text { uthors. } \\
\text { S. This work is } \\
\text { the Creative } \\
\text { ibution License. }\end{array}$ \\
\hline Read online: & \\
\hline 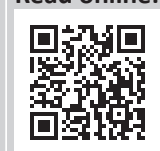 & $\begin{array}{l}\text { Scan this QR } \\
\text { code with your } \\
\text { smart phone or } \\
\text { mobile device } \\
\text { to read online. }\end{array}$ \\
\hline
\end{tabular}

Traces of thoughts. The place of a theology of the Septuagint in biblical scholarship: In the past decades, research has raised the idea of a theology of the Septuagint (LXX) on various occasions. Important works were recently published on this topic in the Handbuch zur Septuaginta and the Septuagint and Cognate Studies series. The general theological tendencies of the LXX are identified by scholars in eschatology, messianism, anti-anthropomorphism and angelology. These tend to all be regarded as further developments of the theology of the Hebrew Bible (HB). However, one can trace the evolution of these and other main topics of the LXX in the New Testament (NT) and in the later apostolic writings as well. Based on three concise case studies, I point out the evolution of theological ideas from the HB through the LXX up to the NT in this paper. First, I will discuss the importance of the 'Name of God'theology which is increasingly present in the LXX and has a key role in the messianic passages of the NT. Then in two points it will be argued that addressing Jesus as kv́pros implies theological accents which can be detected in the LXX. These observations aim to show that the development of the religious ideologies of the HB can be followed not only in the LXX but in the NT and beyond as well. The importance of a theology of the LXX goes beyond the HB research, having significant implications for the NT as well. In fact, such theology could build the bridge between the HB and the NT, and should not only be written from the viewpoint of the HB but also considering the NT.

Contribution: This contribution traces the routes of three theological concepts originating in the Hebrew Bible developed further in the LXX and finally adopted by the New Testament. Based on these three examples, it is suggested here as a novel idea that a theology of the LXX should not be written only with the Hebrew Bible in mind but also considering the New Testament.

Keywords: Theology; Septuagint; Holistic view; New Testament; Name-Theology in the LXX.

In den letzten Jahrzehnten wurde in der Septuaginta-Forschung der Gedanke einer Theologie der Septuaginta (LXX) ${ }^{1}$ aufgeworfen. Gelehrte wie Emanuel Tov (1987), Jan Joosten (2000), Martin Rösel (2006, 2018), Johann Cook $(2010,2017,2018)$ und weitere Experten (Aejmeleaus 2006, Ausloos 2017, Dafni 2009, Douglas 2012, McLay 2010) haben sich zum Thema eines übergreifenden Werkes, das die theologischen Inhalte und Grundaussagen der LXX aufspürt, systematisiert und herausarbeitet, geäußert und die Notwendigkeit und die Grenzen eines solchen Unterfangens unterschiedlich bewertet und herausgestellt. Als Ergebnis dieser intensiven Forschung erscheinen dieser Zeit sehr viele wichtige Beiträge zum Themenkomplex, zum Beispiel die Theologie der LXX in der LXXH Reihe (Ausloos \& Lemmelijn 2020), oder der Konferenzband Toward a Theology of the Septuagint (Cook \& Rösel 2020). Dabei geht es um die 'Gattung' 'Theologie', wie wir sie im Falle der Hebräischen Bibel (HB) kennen, die oft den Titel 'Theologie des AT' trägt. Eine Theologie der LXX soll genau dasselbe leisten, nämlich eine Zusammenschau der großen Themen und deren Entwicklung innerhalb der einzelnen Schriften. Dass die Theologie der LXX für die Erforschung der HB wertvoll ist, steht fest und ist unbestreitbar, jedoch sollte man sich damit nicht begnügen. Heutzutage werden die holistischen, inter- und multidisziplinären Ansätze in allen wissenschaftlichen Diskussionen hochgeschätzt und in unterschiedlichen tiefgreifenden Projekten durchgeführt. Wir leben in einem Zeitalter, in dem zu unzähligen Problemen und Fragen enorm viele Einzelstudien vorliegen, die man wieder in das große Ganze einzubinden hat. In diesem Beitrag möchte ich einen Schritt in diese

1.Dieser Aufsatz geht zurück auf einen Vortrag, den ich am 06.12.2019 auf der II. LXX-Hungarica Konferenz in Klausenburg gehalten habe. Hier bedanke ich mich bei Prof. Siegfried Kreuzer und Prof. Martin Meiser für die wertvollen Anregungen zu diesem Thema. 
Richtung im Bereich der Theologie der LXX machen, indem ich mögliche Traditionslinien und Berührungspunkte nicht nur mit der Theologie der HB, sondern auch mit der Theologie des Neuen Testaments (NT) aufzeige. Davor möchte ich aber einige methodologischen Voraussetzungen eines solchen Ansatzes nennen und die möglichen Problemhorizonte aufzeigen.

\section{Methodologische Voraussetzungen}

$\mathrm{Zu}$ klären ist zuerst, was Theologie als Gattung ist und was sie leisten kann. Klassischerweise wird unter diesem Begriff ein zusammenfassendes Werk verstanden, das auf eine methodisch reflektierte Weise die leitenden Themen und Grundgehalte der einzelnen Schriften des jeweiligen Korpus systematisch aufarbeitet und nach einer vorherbestimmten inneren Logik darstellt. In der neueren exegetischen Forschung wird jedoch vom Gedanken der Theologie als selbstständiger Fachdisziplin zunehmend abgerückt. Stattdessen spricht man von Theologien (so Gerstenberger 2001) oder von der Religionsgeschichte Israels (Albertz 1992). Aber auch unter denen, die es noch wag(t)en, eine 'Theologie' zu schreiben, herrscht keine einheitliche Meinung darüber, was die Mitte der HB sei, welches die Grundlinien, die das Gerüst einer solchen Theologie bilden, seien und ob die $\mathrm{HB}$ ein einheitliches theologisches System überhaupt entwickelt habe (Jeremias 2015a; Levin 2008). Die neueren Ansätze (etwa die von Jeremias 2015b und Schmid 2019) scheinen sich nach den entstehungsgeschichtlichen Gegebenheiten der einzelnen Schriften zu richten und darin ein logisches, chronologisches Prinzip $\mathrm{zu}$ verfolgen. Ähnliches gilt auch für die Theologie des Neuen Testaments (Graf 2011:271-280; Schnelle 2009:49-54).

Was aber die Theologie der LXX anbelangt, wird die oben angewandte Definition problematisch, da die LXX nicht eine selbstständige Größe, sondern eine Übersetzung der HB darstellt, deren Theologie vor allem in der Übersetzungstechnik zum Ausdruck kommt (Ausloos \& Lemmelijn 2020:23). Diese Theologie baut also auf der Theologie der HB auf und kann deshalb in den meisten Fällen bloß als ihre Rezeption und Weiterführung betrachtet werden (Douglas 2012:116; Rösel 2006:242-243; Schmid 2019:\$28). Wie Tov zutreffend schreibt: '[A]lle Elemente in der Übersetzung hängen irgendwie mit der Hebräischen Bibel zusammen. Entweder bilden sie ihr Gegenstück oder sie sind Ergänzungen zu gewissen Elementen der Hebräischen Bibel' (Tov 1987:238; vgl. Joosten 2000:33). Darin erschöpft sich die Theologie der LXX allerdings nicht ganz. Zwar fußt sie stark auf der Theologie der HB, jedoch gibt es Bücher, die nur auf Griechisch existieren, oder solche, die sich nicht nur als einfache Übersetzung ihrer Vorlage wahrnehmen lassen, sondern auch als deren Auslegung und Paraphrase, wie zum Beispiel die LXX Proverbia (s. dazu Cook 1997:323-326). Dies bedeutet, dass man im Fall der LXX zwar mit einer auf den Theologien der HB gründenden Theologie zu tun hat, aber an vielen Stellen lässt sie sich auch als Weiterführung und Bereicherung der Theologie der HB betrachten und sogar als selbstständige Theologie wahrnehmen (Rösel 2006:242).
Da die Theologie der LXX eng mit der der HB zusammenhängt, muss man auf das Verhältnis der beiden näher eingehen.

Die LXX ist die griechische Übersetzung der HB, aber nicht einfach die Übersetzung des masoretischen Textes (MT). Auch in den Fällen, wo die LXX einen dem MT nahstehenden Text übersetzt, muss man auf die Mannigfaltigkeit der Texte achten und darf die Vorgänge nicht allzu einfach und einseitig auffassen. Weder die HB noch die LXX sind einheitliche Größen, sondern von beiden gibt es Varianten, Rezensionen und Revisionen. Überdies scheint die LXX an vielen Stellen einer vom MT abweichenden hebräischen Texttradition $\mathrm{zu}$ folgen (vgl. Ausloos \& Lemmelijn 2020:35-38). Zum Beispiel im Fall des Jeremia weist die LXX einen kürzeren Text auf, der mit einer der Jeremia-Rollen aus Qumran übereinstimmt $\left(4 \mathrm{QJer}^{\mathrm{b}}\right){ }^{2}{ }^{2} \mathrm{Im}$ Fall der Tora stimmt die LXX in 1900 Fällen, in denen sie sich vom Text des MT unterscheidet, mit dem Samaritanischen Pentateuch überein (Douglas 2012:113). Dieses Bild wird noch komplizierter, wenn man in ähnlicher Weise nach dem Textbestand der LXX fragt, die weitere Rezensionen mit eigenen Hintergründen und sogar Parallelübersetzungen hat. Aber auch wenn man nach der ältesten Textform, nach der Ur-LXX (OG) trachtet, muss man damit rechnen, dass sie nicht aus einem Guss stammt, sondern dass verschiedene Übersetzer einzelne Bücher übersetzt haben, deren Umfang nicht mit den buchübergreifenden, ursprünglichen Schriften (Deutronomistisches Geschichtswerk, Priesterschrift, Chronistisches Geschichtswerk usw.) übereinstimmt. Man hat zum Beispiel in der LXX mit einer auf Bücher zersplitterten Rezeption und Wahrnehmung der deutronomistischen (dtn) Theologie zu tun. Was sich im MT als eine 'einheitliche' Linie zeigt, wird in der LXX gar nicht einlinig erscheinen. Das heißt, im Fall der LXX kann man das Konzept der Theologien ${ }^{3}$ nicht umgehen, ganz im Gegenteil, der Gedanke der Pluralitätgehörtzudenmethodischen Grundvoraussetzungen der Beschäftigung mit der LXX.

An den oben aufgezeigten Themenkomplexen knüpft die Frage an, welcher Grundtext als Basis der Untersuchung dienen soll. Soll man die theologischen Grundlinien der LXX aufgrund einer diplomatischen Textedition wie etwa des Vaticanus oder Alexandrinus nachzeichnen (Dafni 2009:447; vgl. McLay 2010), oder sich auf eine eklektische Rekonstruktion der Urseptuaginta stützen (Cook 2017:278; Douglas 2012:110; Rösel 2006), etwa der Göttinger Septuaginta, deren letztes Drittel noch nicht vorliegt? Beide Optionen haben Befürworter und Gegner. Im Idealfall stünden alle Manuskripte zur Verfügung, und wir hätten die Chance, alle Aspekte in einer Theologie unterzubringen. Jedoch muss man damit rechnen, dass vielleicht viele bedeutungsvolle und wirkungsreiche Texte verloren gegangen und nicht mehr rekonstruierbar sind (Ausloos \& Lemmelijn 2020:26-28). Deshalb sollte man die erreichbaren Quellen möglichst vollständig aufarbeiten und in Betracht ziehen und die Analyse komparativ durchführen.

2.In Qumran wurden aber auch als proto- und semimasoretisch eingestufte JeremiaRollen entdeckt (s. Lange 2018:280-302).

3.Dies akzentuiert deutlich Cook (2017:278-279). Vergleiche Ausloos \& Lemmelijn (2020) 
Dementsprechend tendiert die Forschung in einer Theologie der LXX dazu, nicht nur den Basistext, sondern dessen Bezug $\mathrm{zu}$ den wichtigeren Varianten und Rezensionen zu berücksichtigen und einen diachronen Entwurf unter den Textzeugen zu leisten. So wäre die immer auftretende Frage nach den 'ursprünglichen' Lesungen, die gar nicht so leicht zu identifizieren sind, vor allem bei Parallelübersetzungen, zumindest teilweise entschärft, da alle Varianten in irgendeiner Form zu Wort kommen.

Angesichts dieser Schwierigkeiten in Bezug auf die Theologien und den Textbestand der HB und der LXX stellt sich die Frage, wie man die theologischen Facetten der LXX überhaupt sichtbar machen kann. Von der Tradition her sollte ein besonderer Akzent auf die Tora gelegt werden, umso mehr als diese im Übersetzungsprozess der LXX als Muster und sogar als theologisches Wörterbuch für die späteren Übersetzungen fungiert hat (Tov 1987:245). Auf diese Weise wurden viele theologische Äquivalente zwischen den hebräischen und griechischen Termini für die späteren Generationen vorgegeben. Weitere für die LXX kennzeichnende, theologische Merkmale sind an den Stellen zu suchen, wo die LXX mehr, weniger oder anderes als der MT sagt (Aejmeleaus 2006:30; Cook 2010:622; Dafni 2009:453; Joosten 2000:33; Rösel 2006:242; vgl. Ausloos \& Lemmelijn 2020:30-39). Der Ausgangspunkt einer Theologie der LXX soll dementsprechend in den Differenzen zum MT liegen. Freilich ergäbe das noch kein zusammenhängendes System, zumindest bei den Büchern nicht, die der masoretischen Tradition nahestehen. Deshalb bilden das Grundgerüst nach wie vor die Theologien der HB.

Eine Theologie der LXX ist also auf der HB basierend und entlang der Unterschiede zum MT zu entwerfen. Darin liegt aber ein weiteres Problem, wie oben angedeutet, dass wir nicht immer sicher sein können, ob ein Unterschied infolge des Übersetzens entstanden ist oder schon in der Vorlage vorhanden war (Douglas 2012:112) und dadurch nicht die Rezeption des MT, sondern eine andere hebräische Tradition aufzeigt. Wenn man nach den theologischen Weiterführungen und spezifischen Akzenten in der LXX trachtet, muss man in der Lage sein, den Unterschied $\mathrm{zu}$ machen zwischen absichtlichen Änderungen der LXX, anderen Vorlagen oder aus Verlesungen entstandenen Lesarten. Es scheint aber, dass eine gewisse methodische Unsicherheit in dieser Hinsicht weiterhin nicht zu eliminieren sein wird. Um diese und andere Unsicherheiten $\mathrm{zu}$ minimieren, schlug Cook in mehreren Publikationen vor, zuerst tiefgreifende Einzelstudien und Kommentare zu den Büchern der LXX zu verfassen, und erst danach nach dem gemeinsamen theologischen Nenner zu fragen (Cook 2017:279, 2018). In dieser Hinsicht wurde in den letzten Jahrzehnten viel getan (vgl. Cook 2018), und es ist kein Zufall, dass im Jahre 2020 so viele wichtige Publikationen im Bereich der Theologie der LXX erscheinen.

Eine Theologie der LXX verfolgt also die Absicht, die Unterschiede zum MT aufzuzeigen und darin die theologischen Merkmale der LXX zu identifizieren. Dabei steht trotz den methodischen Unsicherheiten eine starke
Bindung der LXX und der HB fest. Von historischem Gesichtspunkt aus betrachtet kann eine legitime Korrelation nicht bezweifelt werden. Aber ist das auch mit dem NT der Fall? In den letzten Jahren wurde der Beziehung der LXX zum NT zunehmende Aufmerksamkeit geschenkt und die Frage der Rezeptionsgeschichte gestellt. ${ }^{4}$ In diesem Bereich sind weitere Studien und Forschungen erwünscht und $\mathrm{zu}$ erwarten, die zu einem besseren Verständnis der Wandlung theologischer Konzepte über Jahrhunderte hinweg beitragen können. Im Folgenden möchte dieser Beitrag in diese Richtung einen weiteren Schritt machen.

\section{Die LXX und das Neue Testament}

In der Frühkirche wurde die LXX gegenüber der HB wegen einiger wichtiger, von dem MT abweichender Lesarten bevorzugt. ${ }^{5}$ Ein Paradebeispiel dafür ist Jesaja 7:14, wo im MT nur von einer jungen Frau (עָלְָָָה) die Rede ist, während die LXX von einer Jungfrau ( $\pi \alpha \rho \theta \varepsilon \dot{v}$ o $)$ spricht. Diese letzte Interpretation wurde verständlicherweise im Lichte der Evangelien stark rezipiert, und für die Priorität der griechischen Übersetzung eingesetzt (Müller 1993:195). So hat die LXX in der alten Kirche ein hohes Ansehen erreicht und wurde bis heute zur Bibel der Orthodoxen Kirchen weltweit. Von der Tradition her wäre also eine Verknüpfung keineswegs verfehlt, sogar erwünscht. Wenn man aber auf eine methodisch reflektierte Weise die beiden Korpora verbinden möchte, muss man nach direkten und indirekten Traditionslinien fragen und die Verbindung mit Hilfe dieser Berührungspunkte herstellen.

Es gab auch im 20. Jahrhundert schon Versuche, die Beziehung der LXX zum NT wissenschaftlich, vor allem sprachwissenschaftlich und ideologisch zu erklären. Ein markanter Vertreter dieser Richtung war Bertram, der in der LXX die preparatio evangelica sah und, auch anderen Gelehrten folgend, von der LXX-Frömmigkeit sprach (vgl. Bertram 1957:225-249, 1961:1707-1709). Nach Bertram sei die LXX eine einheitliche theologische Größe, repräsentativ für das ganze hellenisierte Diaspora-Judentum, die viele Begriffe ähnlich oder gleich dem NT gebrauche und am Anfang einer Entwicklung stehe, die ihren Höhenpunkt im NT erreiche. Von einem solchen vereinfachten Bild der LXX und des Diaspora-Judentums wurde in der Forschung mit Recht Abstand genommen (s. die Kritik bei Rösel 2006: 239-241). Freilich ist, wie die LXX und die HB, auch das NT nicht monolithisch, sondern hat selbst einen längeren Entstehungsprozess durchlaufen. In diesem Prozess hat aber auch die LXX eine bestimmte Rolle gespielt, die sowohl von der LXX als auch vom NT her beleuchtet werden kann.

Von der LXX herkommend darf man nicht vergessen, dass der Begriff Siebzig für die gesamte Übersetzung der HB erst in der christlichen Ära, ab der Zeit Justin des Märtyrers († 165 n. Chr., vgl. Dialog mit dem Juden Tryphon LXVIII; LXXILXXII; CXXXI; CXXXVII) angewandt wurde (Meade 2018).

4.Ohne Anspruch auf Vollständigkeit (vgl. McLay 2003; Law 2013; die Aufsätze in Bons 2014 und Kraus 2014).

5.Zu diesem Thema siehe Müller (1993, 1996). 
Der Aristeasbrief, der die Ursprungsgeschichte der griechischen Übersetzung der HB enthält, spricht nur von der Tora und nennt 72 Übersetzer (je 6 aus den 12 Stämmen). Dieser pseudepigrafe Briefroman stammt wahrscheinlich aus der 2. Hälfte des 2. Jahrhunderts vor Christus (Müller 1993:200; Tilly 2007; vgl. Cook 2018), also aus einer Zeit, in der sicher schon andere Schriften der HB übersetzt vorlagen. ${ }^{6}$ Die Zahl Siebzig als Zahl der Übersetzer der Tora, wird erstmals bei Josephus vorgefunden, der wahrscheinlich die Zahl 72 mit der stärkeren und wirkungsreicheren 70 ersetzt, wobei er die Herkunft und die Zahl der Übersetzer nach den Stämmen offensichtlich kennt (Major 2013:18). Diese Tradition greifen die Kirchenväter auf und weiten sie auf die gesamte griechische Übersetzung der HB aus. Das bedeutet, dass wenn man von der LXX im Sinne einer Sammlung von Schriften spricht, die über die Tora hinausgeht, man schon in einer kirchlichen Tradition steht.

Hinzu kommt, dass die Bücher, die heute als LXX bezeichnet werden, in christlichen Codices aus dem 4-5. Jahrhundert vor Christus erhalten sind. Daraus ergibt sich, dass das Konzept der LXX, das wir oft selbstverständlich anwenden, im Gebrauch der alten Kirche verwurzelt ist. Nach dem Aristeasbrief hingegen, das heißt, nach dem inneren Zeugnis des hellenisierten Judentums, bezieht sich die Zweiundsiebzig nur auf die fünf Bücher Mose, und die haben einen unbestreitbaren Vorrang den anderen Büchern gegenüber.

Ist demzufolge die Theologie einer LXX, die mehr Bücher als die Tora enthält, schon von vornherein eine kirchliche Theologie, oder soll sich eine Theologie der LXX gemäß der Vorstellung des Aristeasbriefes nur auf die griechische Tora konzentrieren? Die Antwort auf diese Frage kann nicht als Entweder-oder angegeben werden. Obwohl die LXXCodices in christlichen Handschriften erhalten blieben, kann daraus nicht geschlossen werden, dass das Christentum den 'Kanon' der LXX erfunden hat. Ganz bestimmt haben die Kirchenväter eine vorgegebene jüdische Tradition übernommen, die aber vor allem nicht in Palästina entstanden war, sondern in der Diaspora, möglicherweise hauptsächlich in Ägypten. Wie das aus den Listen deutlich wird, wurden in der Diaspora nicht nur jene Bücher des hebräischen Pendants weitertradiert, sondern auch weitere, die auf Hebräisch gar nicht existierten. ${ }^{7}$ Gab es da einen alexandrinischen Kanon? Auf eine gewisse Weise ja, aber nur in dem Sinne, dass diese Bücher als 'erbauliche' religiöse Literatur, als Inspirationsquelle für das Diaspora-Judentum fungiert haben, ohne aber als 'heiliger Text' angesehen $\mathrm{zu}$ werden (vgl. Joosten 2016; Schaper 1999:96). Jedoch war, wie es aus dem Aristeasbrief deutlich hervorgeht, auch dieser 'Kanon' in seiner Zeit Kontroversen ausgesetzt.

6.Aristeasbrief 30 (zitiert nach Rießler 1928:197): "Es fehlen aber noch mit wenigen anderen die Bücher des jüdischen Gesetzes. Sie sind ja in hebräischer Schrift und Sprache abgefaßt; sie wurden aber recht ungenau und mit Abweichungen vom ursprünglichen Text übersetzt, wie die Kenner berichten. Es fehlte ihnen ja an der HB hinweisen, die aber hier bewusst zurückgedrängt werden (vgl. Douglas 2012:108).

7.Notabene. Im Vaticanus fehlen die Makkabäerbücher, im Sinaiticus das 2. und 3. Makkabäerbuch, der Alexandrinus hat dagegen alle Makkabäerbücher sowie das Buch der 14 Oden (mit dem Gebet des Manasse) (vgl. Schöpflin 2009).
Nichtdestotrotz weist die weitere Sammlung der LXX und ihre Codices auf die kirchliche Tradition hinter dem Konzept, das zwar wahrscheinlich eine jüdische Anschauung aufgreift, aber gleichzeitig die Wichtigkeit der LXX für die alte Kirche und indirekt für das NT herausstellt.

Es gibt indes nicht nur indirekte Berührungspunkte zwischen der LXX und dem NT, sondern sogar elementare, die man in den Zitaten erfassen kann. Das NT scheint, wenn es die HB zitiert, überwiegend auf die LXX zurückzugreifen (Koch 1986:48-57; Menken 1996, 2004; Scott 1995:132-134). Dies lag auf der Hand, da die Sprache der apostolischen Kirche das Griechische war, und die ins Griechische übersetzten Bücher der HB sprachlich leichter zugänglich waren als die hebräischen Originale. Dabei ist sicherlich auch die Kenntnis des MT anzunehmen. Trotzdem kann in den Fällen, wo die Zitate dem MT näherstehen, nicht vorausgesetzt werden, dass das NT 'eigenständig auf den hebräischen Wortlaut der Schrift zurückgreift, sondern an diesen Stellen eine dem H[ebräischen] T[ext] angenäherte [LXX] Vorlage verwendet' (Koch 1986:57). ${ }^{8}$ Das impliziert keine theologische Bevorzugung des griechischen Textes zulasten der hebräischen Version, sondern eine praktische Überlegung: Statt Textpassagen frei zu übersetzen, war es leichter, eine Übertragung mit gewisser Tradition zu übernehmen und in den eigenen griechischen Text einfließen zu lassen. Damit wird gleichzeitig die LXX der Vermittlerkanal von Inhalten der HB. Man könnte etwas vereinfacht formulieren, dass die Theologie der HB im NT durch die LXX rezipiert wird. Auf der Suche nach maßgebenden Themen der LXX konnte man sich deshalb nicht nur an den vorgegebenen Traditionen im Alten Testament (AT), sondern auch an deren neutestamentlicher Rezeption orientieren. Schon das Genre 'Theologie' gibt uns diese Freiheit. Nach Jeremias unterscheidet sich eine 'Theologie' eben darin von einer 'Religionsgeschichte', dass letztere 'das Werden des biblischen Glaubens über die Jahrhunderte seit seiner Entstehung' beschreibt, während sich 'eine Theologie des AT [...] auf die Darstellung des bleibend Gültigen [konzentriert]' (Jeremias 2015a:50).

Was dieses bleibend Gültige sein soll, zeigt uns auch die Rezeptionsgeschichte, indem man die HB, die LXX und das NT auf eine Entwicklungslinie platziert. Allerdings muss man sich dessen auch bewusst sein, dass diese Schriften nicht das ganze theologische Denken repräsentieren und abbilden. Viele Traditionen, die nicht verschriftlicht oder absichtlich zurückgedrängt wurden, oder nur für marginale Sozialgruppen von Belang waren, sind nicht erhalten und haben trotzdem eine wichtige Rolle in der Ausformulierung der kanonischen Konzepte gespielt, die wir heute nur sehr vage - wenn überhaupt - rekonstruieren können. Wir haben nicht alle Teile des Puzzles, aber wir wissen ja, dass alle Teile, die wir haben, zu demselben Bild gehören. An manchen Stellen können wir jedoch leicht einen Zusammenhang aufdecken und den Lauf des theologischen Denkens nachzeichnen.

8.Koch 1986:57. In dem Kontext geht es um den Schriftgebrauch des Paulus, aber in der 4. Fußnote weitet Koch diese Sicht auf das gesamte NT aus. Siehe auch Menken

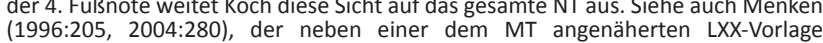
voraussetzt, dass Johannes in 12:40 und 13:18 den MT selber übersetzt hat. 
Im Folgenden möchte ich drei solche Konzepte und deren Wandlung darstellen, die an den Umgang mit dem Namen Jhwhs anknüpfen und so grundlegende gemeinsame Denkschemata zwischen der LXX und dem NT aufdecken, mit der Absicht, zu weiteren Studien in diese Richtung anzuregen.

\section{Theologumena in der LXX und im Neuen Testament}

Die meisten Forscher heben als allgemeine theologische Tendenzen der LXX folgende Aspekte hervor: Eschatologie, Messianismus, Anti-Anthropomorphismus (Rösel 2006, Douglas 2012, Cook 2017), Angelologie (Dafni 2009:450-451). ${ }^{9}$ Das sind allesamt Themen, die das Rückgrat des NT bilden und die neutestamentliche Theologie stark prägen. Diese sind auf Schritt und Tritt anzutreffen, nicht nur in den Zitatpassagen. Aber darin erschöpfen sich die gemeinsamen theologischen Züge bei Weitem nicht.

Rösel macht in seinen Arbeiten darauf aufmerksam, dass die Name-Gottes-Theologie (Schem-Theologie) in der LXX mehr und mehr an Bedeutung gewinnt (Rösel 1998:55-60, 2006:246-247). Ein Paradebeispiel dafür ist Levitikus 24:16, wo nicht nur derjenige hingerichtet werden soll, der den

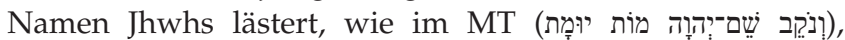

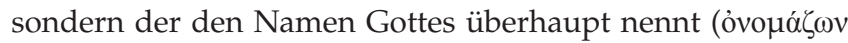

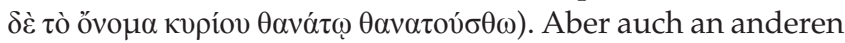
Stellen ist zu beobachten, dass in der LXX der Name Jhwhs auftaucht, wo er in der HB fehlt oder mit einem anderen Begriff ersetzt wird: Numeri 14:21; Deuteronomium 12:26; 17:8,10; Buch der Psalmen 44:27 und so weiter. Es ist weniger wahrscheinlich, alle diese Unterschiede bei so verschiedenen Schriften mit einer abweichenden Vorlage zu erklären, als hier die Tätigkeit der jeweiligen LXX-Übersetzer zu erkennen, die ihren Büchern einen bestimmten theologischen und ideologischen Akzent verleihen wollten und dadurch das ursprünglich dtn. Konzept verstärkt haben (Kató 2019:223).

Die Name-Gottes-Theologie spielt aber nicht nur in der deuteronomischen Theologie und dann in der LXX eine herausragende Rolle, sondern auch im NT lässt sich eine zunehmende Bedeutung der Namenstheologie wahrnehmen (Coutts 2017:26-28; Lee 2005:77-84; Schreiner 2008:245-246). Im Namen Jesu wird getauft (Apg 2:38; 10:48 usw.), geheilt (Apg 3:6,16; 4:10) und es werden Dämonen (Apg 16:8) ausgetrieben, die Menschen sollen sogar durch diesen Namen gerettet werden (Apg 4:12). In der johanneischen Theologie wird der Name Gottes Jesus gegeben (Joh 17:12). Die Jünger sollen (Joh 16:24-26) Dinge von dem Vater in seinem Namen erbitten. An sehr vielen Stellen ist der Name Jesu idiomatischer Vertreter seiner Macht und wirksamen Kraft, ein Symbol für Christus selbst. Das funktioniert ganz analog zu der Schem-Theologie in der HB und darf als ihre Weiterführung betrachtet werden (Grillmeier 1975:41-42; Schreiner 2008:297), indem die LXX eine Zwischenstufe darstellt.

9.Freilich könnte diese Liste noch mit anderen Themen erweitert werden, da die einzelnen Bücher und Schriftkomplexe auch innerhalb der LXX ihre spezifischen theologischen Akzente haben (vgl. Ausloos \& Lemmelijn 2020). Hier richte ich mich jedoch nach den buchübergreifenden Phänomenen.
An den Jhwh-Namen schließt sich noch eine weitere Beobachtung an. In den unterschiedlichen Manuskripten der LXX-Bücher findet man unterschiedliche Lösungen für die Wiedergabe des Tetragramms. In manchen Manuskripten findet sich eine Lücke an der entsprechenden Stelle (Papyrus Rylands Greek 458), in manchen findet sich das Tetragramm in hebräischer Quadratschrift (Papyrus Fouad 266) oder in paläohebräischen Schriftzeichen (Nahal Hever; Oxyrhynchus Papyrus 3522) wieder, und in anderen ist der Gottesname transkribiert (IA $\Omega$ - 4QLXXLevb), oder die hebräischen Buchstaben werden grafisch repräsentiert (ПІПI steht für יהוה - Symmachus, Aquila) (Rösel 2007; Wilkinson 2015:48-88). In vielen Codices wird der Gottesname aber mit кúpı Die Forschung tendiert dazu, in diesem letzten Phänomen die älteste Strategie für die Wiedergabe des Tetragramms zu sehen, während alle anderen Lösungen spätere (jüdische) Revisionen darstellen (Rösel 2007; Wilkinson 2015:48-88 mit weiterer Literatur). ${ }^{10}$ So steht die Bezeichnung 'Herr' für den Namen Gottes in der LXX und hat wahrscheinlich auch Einfluss auf die hebräische Praxis, anstelle des Tetragramms 'Adonai' zu sprechen und in der masoretischen Tradition das Tetragramm entsprechend zu punktieren. ${ }^{11}$ Treffen diese Annahmen $\mathrm{zu}$, dann ist der Befund des NT nicht ohne Belang, dass Jesus als кúpıos bezeichnet und damit in die Nähe Gottes gerückt wird. Freilich dürfen nicht alle Belege als christologische Bekenntnisse aufgefasst werden, da es in vielen Fällen 'nur' um eine Höflichkeitsformel geht. Aber an vielen Stellen geht der Kyriostitel für Jesus über diese Grenzen hinaus, und damit wird ausgedrückt, dass Jesus über alle Oberherren kúpros ist, und 'alle kyrioi durch Christus überwunden sind' (Hörster 2007:108). Diese Aussage hat im 1. Jahrhundert vor Christus, in einer Ära der christlichen Sklaverei, einen bestimmten Nebenklang, den wir nicht überhören dürfen. Darüber hinaus wurzelt die Kyriostitulatur Jesu in dem Auferstehungsglauben und wird im Sinne einer Gottesprädikation gebraucht (1 Kor 8:6; 16:22; Phil 2:11) (Rösel 2000:223-225; Rüggemeier 2017:408-419). So fußt eine theologische Grundaussage des NT auf dem Gebrauch von kúpıos in der LXX.

Möglicherweise ist es auch kein Zufall, dass die Gottesprädikation Jesu eher mit кúpı ऽ und seltener mit $\theta \varepsilon o ́ \varsigma$ (vgl. Joh 20:18) zum Ausdruck gebracht wird. Wie das an manchen Stellen der LXX, bei Philo und in der rabbinischen Literatur ersichtlich ist, wird das Tetragramm eher mit einem gnädigen und erbarmenden Gott assoziiert, während unter אֶלֶהים der strafende Gott vorgestellt wird (Dahl \& Segal 1978; Rösel 2006:245, 2007:423). Dementsprechend hat $\theta$ \&ó $\varsigma$ Er getötet (Gen 38:7); Sodom und Gomorra vernichtet (Gen 13:10) und die Welt durch die Sintflut $\mathrm{zu}$ vernichten beabsichtigt (Gen 6:6-7), während im MT an all diesen Stellen das Tetragramm zu lesen ist. Auf diese Weise, wenn das NT mit кúpı̊ৎ die Göttlichkeit Jesu bekennt, ruft es das Bild des gnädigen Gottes in Erinnerung, der sich für Israel einsetzt, und nicht eines strafenden, rachsüchtigen Demiurgen. Der

10.Wilkinson plädiert für die Idee, dass die Wiedergabe nicht einheitlich sein musste und man mit Parallelphänomenen rechnen darf. Zu anderen Sichtweisen siehe Tov (2004:298)

11.Siehe die Diskussion bei Tov (1987:247 Anm. 6). 
Gedanke, Jesus als das barmherzige Ebenbild Gottes anzusehen, ist auf Schritt und Tritt in den Evangelien und anderen Schriften des NT zu verfolgen und ist ein elementarer Bestandteil der neutestamentlichen Theologie.

Diese Beispiele verdienen deshalb Aufmerksamkeit, da sie keine isolierten Phänomene sind, sondern tragende theologische Grundzüge der beiden Sammlungen. Der Umgang mit dem Namen Jhwhs lässt sich in der LXX nicht auf einige Bücher eingrenzen, sondern ist in allen Schriftteilen zu beobachten. Darin zeigt sich eine wichtige Haltung der Gemeinschaft Gott gegenüber: sie unterstellt sich Gott bedingungslos. Jhwh ist transzendent, wohnt im Himmel, sogar in seinem Namen ist er unbegreiflich und unzugänglich. Aus dieser 'Gotteslehre' erwächst das Bild eines Gottes, der mit den Menschen nur durch seine Engel kommuniziert (Angelologie) und mit den Menschen nicht verglichen werden kann (Anti-Anthropomorphismus). Alle diese Vorstellungen lassen sich reibungslos in das Inventar der schon identifizierten theologischen Charakteristika der LXX einbinden. Aber die Christologie des NT führt genau diese Aspekte weiter. Gott ist auch im NT transzendent, und Jesus hat eben diese enorme Distanz zu überbrücken, da er 'das Ebenbild des unsichtbaren Gottes' (Kol 1:15) darstellt. Das Problem das die Gegner mit Jesus im Johannesevangelium haben, ist eben, dass er im Gegensatz zum erwarteten Messias greifbar ist - seine Herkunft ist bekannt (7:27). Er ist nicht so transzendent, wie er nach der Meinung seiner Gegner sein sollte. Darum bemüht sich das NT, Jesus dem Gott Israels anzunähern, indem die Kraft und Heiligkeit seines und des Namens Jhwhs gleichgesetzt werden. Dabei sind die theologischen Gemeinsamkeiten der LXX und des NT unverkennbar und reizen zur weiteren Forschung an.

\section{Fazit}

Die dargestellten Beispiele werfen etwas Licht auf die enge theologische Beziehung der LXX zum NT, aber es könnten sich viele weitere Fälle anführen lassen, die einen Zusammenhang sichtbar machen. Entlang diesen Zusammenhängen könnte man den Lauf des theologischen Denkens jüdischen Ursprungs zusammenfassen und nachzeichnen und man hätte damit eine holistische Gesamtaufnahme der Theologie in der Hand. Eben die Theologie als Gattung wäre dafür der beste Kandidat. Sie systematisiert nicht nur die Ideologie eines Schriftkorpus, sondern versucht dabei immer, eine holistische Herangehensweise $\mathrm{zu}$ implizieren und immer nach der Einbindung des jeweiligen Details in das Gesamtbild zu fragen. Freilich würde auch dieses Gesamtbild an manchen Stellen unscharf oder ganz dunkel bleiben, da die HB, die LXX und das NT nur hinterlassene Spuren des theologischen Denkens sind, und nicht alle Aspekte ihrer Zeit repräsentieren können. Viele Fragen, was die Quellen, Querbezüge, Hintergründe anbelangt, bleiben in der Schwebe, jedoch hat die Theologie die Aufgabe, aus diesen Spuren einen Weg zu rekonstruieren und das 'bleibend Gültige' in der Theologiegeschichte in seiner ganzen
Reichweite zu verfolgen. Die Theologie der LXX kann also für ein solches Unterfangen einen guten Ausgangspunkt liefern, und leicht von der HB abgeleitet und unkompliziert in die Richtung NT weitergeführt werden. Deshalb könnte die Theologie der LXX der archimedische Punkt der biblischen Theologie sein, der die HB und das NT auf eine wissenschaftlich begründete Weise wieder verbindet und sie zu einem holistischen Bild zusammenfügt. So sollte man eine Theologie der Septuaginta nicht nur von der Hebräischen Bibel her, sondern auch auf das Neue Testament hin entwerfen.

\section{Danksagung}

Ich möchte mich bedanken bei Prof. Renate Klein für das Korrekturlesen des deutschen Textes.

\section{Konkurierende Interessen}

Der Verfasser erklärt hiermit, daß er keinerlei finanzielle oder persönliche Bindung hat, die ihn zweckwidrig beim Schreiben dieses Artikels beeinflusste.

\section{Autorenbeiträge}

Sz.F.K ist der alleinige Autor dieses Forschungsartikels.

\section{Ethische Überlegungen}

Dieser Artikel beachtete alle ethischen Maßstäbe für die Forschung ohne jegliche Berührung mit menschlichen Subjekten.

\section{Finanzierung}

This research is part of the project Prophetic Studies of Prof. Alphonso Groenewald in the Department of Old Testament and Hebrew Scriptures, Faculty of Theology and Religion, University of Pretoria.

\section{Erklärung zur Datenverfügbarkeit}

Die gemeinsame Nutzung von Daten gilt nicht für diesen Artikel, da in dieser Studie keine neuen Daten erstellt oder analysiert wurden.

\section{Haftungsausschluss}

Die in diesem Artikel geäußerten Ansichten und Meinungen sind die des Autoren und spiegeln nicht unbedingt die offizielle Meinung oder Position einer angegliederten Agentur wider.

\section{Literaturverzeichnis}

Aejmeleaus, A., 2006, 'Von Sprache zur Theologie, Methodologische Überlegung zur Theologie der Septuaginta', in M. Knibb (Hrsg.), The Septuagint and Messianism, Bibliotheca Ephemeridum Theologicarum Lovaniensium 195, S. 21-48, Leuven University Press, Leuven.

Albertz, R., 1992, 'Religionsgeschichte Israels in alttestamentlicher Zeit', Grundrisse zum Alten Testament 8, 1-2, Vandenhoeck \& Ruprecht, Göttingen.

Ausloos, H., 2017, 'Sept défis posés à une théologie de la Septante', in L.C Jonker, G.R. Kotzé \& C.M. Maier (Hrsg.), Congress Volume Stellenbosch 2016, Vetus Testamentum Supplements 177, S. 228-250, Brill, Leiden. 
Ausloos, H. \& Lemmelijn, B., 2020, 'Die Theologie der Septuaginta', Handbuch zur Septuaginta 5, Gütersloher Verlagshaus, Gütersloh.

Bertram, G., 1957, 'Praeparatio evangelica in der Septuaginta', Vetus Testamentum 7, 225-249. https://doi.org/10.1163/156853357X00197

Bertram, G., 1961, 'Septuaginta-Frömmigkeit', Religion in Geschichte und Gegenwart 5, 1707-1709.

Bons, E. (Hrsg.), 2014, 'The reception of Septuagint words in Jewish-Hellenistic and Christian Literature', Wissenschaftliche Untersuchungen zum Neuen Testament 367, Mohr Siebeck, Tübingen.

Cook, J., 1997, 'The Septuagint of Proverbs', Vetus Testamentum Supplements 69, Brill, Leiden.

Cook, J., 2010, 'Towards the formulation of a theology of the Septuagint', in A. Lemaire (Hrsg.), Congress volume of IOSOT Ljubljana 2007, Vetus Testamentum Supplements 133, pp. 621-640, Brill, Leiden.

Cook, J., 2017, 'A theology of the Septuagint?', Festschrift for Sakkie Spangenberg, Old Testament Essays 30(2), 265-282. https://doi.org/10.17159/2312-3621/2017/ v30n $2 \mathrm{a} 5$

Cook, J., 2018, 'Recent developments in Septuagint research', HTS Teologiese Studies/ Theological Studies 74(3), 5002. https://doi.org/10.4102/hts.v74i3.5002

Cook, J. \& Rösel, M., 2018, Toward a theology of the Septuagint: Stellenbosch congress on the Septuagint, 2018, SBL, Atlanta, GA.

Coutts, J.F., 2017, 'The divine name in the Gospel of John', Wissenschaftliche Untersuchungen zum Neuen Testament 2/447, Mohr Siebeck, Tübingen.

Dafni, E., 2009, 'Theologie der Sprache der Septuaginta im Horizont des Altgriechischen Schrifttums und Denkens', Journal for Semitics 18(2), 434-457.

Dahl N.A. \& Segal A.F., 1978, 'Philo and the Rabbis on the Names of God', Journal for the Study of Judaism 9, 1-28.

Douglas, A., 2012, 'Limitations to writing a theology of the Septuagint', Journal of Septuagint and Cognate Studies 45, 104-117.

Gerstenberger, E.S., 2001, Theologien im Alten Testament, Kohlhammer, Stuttgart.

Graf, D., 2011, Unterwegs zu einer Biblischen Theologie, V \& R unipress, Göttingen.

Grillmeier, A., 1975, Christ in Christian Tradition, John Knox Press, Atlanta, GA.

Hörster, G., 2007, Theologie des Neuen Testaments, TVG, Wuppertal.

Jeremias, J., 2015a, 'Hauptprobleme einer Theologie des Alten Testaments', in F. Hartenstein \& J. Krispenz (eds.), Studien zur Theologie des Alten Testaments, S. 47-64, Mohr Siebeck, Tübingen. (Forschungen zum Alten Testament 99) $=$ Balogh Cs. (Hrsg.), Testimony, dispute, advocacy, pp. 15-33, Exit Kiadó, Kolozsvár, 2012.

Jeremias, J., 2015b, 'Die Theologie des Alten Testaments', Grundrisse zum Alten Testament 6, Vandenhoeck \& Ruprecht, Göttingen.

Joosten, J., 2000, 'Une théologie de la Septante? Réflexions méthodologiques sur l'interprétation de la version grecque', Revue de Théologie et de Philosophie 132(1), 31-46.

Joosten, J., 2016, 'The origin of the Septuagint Canon', in S. Kreuzer, M. Meiser \& M. Sigismund (Hrsg.), Die Septuaginta - Orte und Intentionen, S. Wissenschaftliche M. Sigismund (Hrsg.), Die Septuaginta-Orte und Intentionen, S. Wissenschaftliche
Untersuchungen zum Neuen Testament 361,688-699, Mohr Siebeck, Tübingen.

Kató, S.F., 2019, 'Der Tempelweihspruch Salomos (I Reg 8,12-13/LXX III Bas 8,53). Ein neuer Vorschlag', Zeitschrift für die alttestamentliche Wissenschaft 131(2) 220-234. https://doi.org/10.1515/zaw-2019-2004

Koch, A.D., 1986, 'Die Schrift als Zeuge des Evangeliums', Beiträge zur Historischen Theologie 69, Mohr Siebeck, Tübingen.

Kraus, W. (Hrsg.), 2014, Die Septuaginta - Text, Wirkung, Rezeption: 4. Internationale Fachtagung veranstaltet von Septuaginta Deutsch (LXX.D), Mohr Siebeck Tübingen.

Lange, A., 2018, 'Texts of Jeremiah in the Qumran Library', in J.R. Lundblom, C.A. Evans \& B.A. Anderson (Hrsg.), The Book of Jeremiah, Vetus Testamentum Supplements 178, S. 280-302, Brill, Leiden.

Law, T.M., 2013, When God spoke Greek: The Septuagint and the making of the Christian Bible, Oxford University Press, New York, NY.

Lee, A.H.I., 2005, 'From Messiah to pre-existent Son', Wissenschaftliche Untersuchungen zum Neuen Testament 2/192, Mohr Siebeck, Tübingen.
Levin, C., 2008, 'Das Alte Testament auf dem Weg zu seiner Theologie', Zeitschrift für die Theologie und Kirche 105(2), 125-145. https://doi.org/10.1628/ 004435408784514436

Major, T., 2013, 'The number seventy-two: Biblical and Hellenistic beginnings to the early middle ages', Sacris Erudiri 52, 7-45. https://doi.org/10.1484/J.SE.1.103821

McLay, T., 2003, The use of the Septuagint in New Testament research, Eerdmans, Grand Rapids, MI.

McLay, T., 2010, 'Why not a theology of the Septuagint?', in W. Kraus, M. Karrer \& M. Meiser (Hrsg.), Die Septuaginta: Texte, Theologien, Einflüsse, Wissenschaftliche Untersuchungen zum Neuen Testament 252, S. 607-620, Mohr Siebeck, Tübingen.

Meade, J.D., 2018, 'Was there a "Septuagint Canon"?', Didaktikos 1(3), 40-42.

Menken, M.J.J., 1996, 'Old Testament quotations in the fourth Gospel', Contributions to Biblical Exegesis \& Theology 15, Pharos, Kampen.

Menken, M.J.J., 2004, 'Matthew's Bible: The Old Testament Text of the Evangelist', Bibliotheca Ephemeridum Theologicarum Lovaniensium 173, Leuven University Press, Peeters, Leuven.

Müller, M., 1993, 'The Septuagint as the Bible of the New Testament Church', Scandinavian Journal of the Old Testament 7(2), 194-207. https://doi. org/10.1080/09018329308585017

Müller, M., 1996, 'The first Bible of the church', Journal for the Study of the Old Testament, Supplement Series 206, Sheffield Academic Press, Sheffield.

Rießler, P., 1928, Altjüdisches Schrifttum außerhalb der Bibel, Filser, Augsburg.

Rösel, M., 1998, 'Theo-Logie der griechischen Bibel. Zur Wiedergabe der Gottesaussagen im LXX Pentateuch', Vetus Testamentum 48(1), 49-62. https:// doi.org/10.1163/1568533982721910

Rösel, M., 2000, 'Adonaj, warum Gott “Herr" genannt wird', Forschungen zum Alten Testament 29, Mohr Siebeck, Tübingen.

Rösel, M., 2006, 'Towards a "Theology of the Septuagint"', in W. Kraus \& R.G. Wooden (Hrsg.), Septuagint research: Issues and challenges in the study of the Greek Jewish scriptures, Septuagint and Cognate Studies 53, S. 239-252, SBL, Atlanta, GA.

Rösel, M., 2007, 'The reading and translation of the divine name in the Masoretic tradition and the Greek Pentateuch', Journal for the Study of the Old Testament 31(4), 411-437. https://doi.org/10.1177/0309089207080558

Rösel, M., 2018, 'Eine Theologie der Septuaginta? Präzisierungen und Pointierungen', in F. Ueberschaer, T. Wagner \& J.M. Robker (Hrsg.), Theologie und Textgeschichte: Septuaginta und Masoretischer Text als Äußerungen theologischer Reflexion, Septuaginta und Masoretischer Text als Außerungen theologischer Reflexion,
Wissenschaftliche Untersuchungen zum Neuen Testament 407, S. 1-35, Mohr, Wissenschaftliche
Siebeck, Tübingen.

Rüggemeier, J., 2017, 'Poetik der markinischen Christologie', Wissenschaftliche Untersuchungen zum Neuen Testament 458, Mohr Siebeck, Tübingen.

Schaper, J., 1999, 'The Rabbinic Canon and the Old Testament of the early church: A social-historical view', in A. Van der Kooij \& K. Van der Toorn (Hrsg.), Canonization and Decanonization, pp. 94-106, Brill, Leiden.

Schmid, K., 2019, A historical theology of the Hebrew Bible, Eerdmans, Grand Rapids, MI. Schnelle, U., 2009, Theology of the New Testament, Baker Academic, Grand Rapids, MI.

Schöpflin, S.K., 2009, 'Kanon', wibilex, viewed 02 February 2020, from https://www. bibelwissenschaft.de/fileadmin/buh_bibelmodul/media/wibi/pdf/Kanon_ AT 2019-05-29_13_27.pdf.

Schreiner, T., 2008, New Testament theology, Baker Academic, Grand Rapids, MI.

Scott, J.J., Jr., 1995, Jewish backgrounds of the New Testament, Baker Academic, Grand Rapids, MI.

Tilly, M., 2007, 'Aristeasbrief', wibilex, viewed 15 January 2020, from https://www. bibelwissenschaft.de/fileadmin/buh_bibelmodul/media/wibi/pdf/ Aristeasbrief_2018-09-20_06_20.pdf.

Tov, E., 1987, 'Die Septuaginta in ihrem Theologischen und Traditionsgeschichtlichen Verhältnis zur Hebräischen Bibel', Judaica et Christiana 11, 237-265.

Tov, E., 2004, Scribal practices and approaches reflected in the texts found in the Judean Desert, Brill, Leiden.

Wilkinson, R.J., 2015, 'Tetragrammaton: Western Christians and the Hebrew Name of God', Studies in the History of Christian Traditions 179, Brill, Leiden. 